\title{
Image Analysis on Heart Rate Variability Based on Curve Fitting in Computer
}

\author{
Guofeng Liu, Xiaoqin Yin \\ Sports Training Department of Physical Education College, Shihezi University, Shihezi, China \\ liu_guo_feng3@126.com
}

Keywords: time domain; frequency domain; nonlinear analysis; ap model; heart rate variability; equilibrium;

\begin{abstract}
As the ancient Chinese literature search the effective combination of martial arts and fitness exercises, Taichi in the mass has been widely promoted and learning. In these years heart rate variability is generally affected by the physiology of the key areas of concern, therefore this article aims at the development of Taichi in college students were investigated by experiment, experiment and statistics analysis, to explore the Taichi special exercise on heart rate variability, in order to better the shadowboxing for promotion and practice. The research indicated that, the Taichi sport can effectively reduce the heart rate variability ( HRV ), while long-term Taichi sport can improve the recovery rate and heart rate variability in a calm state of autonomic vagus tension level, but also can effectively be vegetative nervous system equilibrium level of ascension, the to strengthen the Taichi sport promotion and study has certain practical significance.
\end{abstract}

\section{Introduction}

In the current people's material can meet the requirement of times, pay more attention to the pursuit of spirit, people gradually realized that the quality of life and the significance of importance, not only to maintain longevity, but also pay attention to the meaning of life, quality level. Taichi is a very popular people's favorite sport, which is a collection of Chinese martial arts and modern aerobics a fitness boxing. Because it is the main focus on some flower, unlike basketball or soccer, strenuous exercise, so also in people of all ages have been widely spread. Heart rate variability is the HRV, which mainly reflects in a certain period of time between the sinus cycle changes a phenomenon, is mainly used to evaluate the sympathetic and parasympathetic nervous tension and mutual balance. Because the heart rate variability and blood vessels of the human body between the activities of information the existence of a great relationship, so in recent years has also been extensive academic research, but also in the sports community has been applied widely. The article is mainly aimed at the development of Taichi in the investigation, comparison, analysis of Tai Chi exercise on heart rate variability influence situation, in order to guide the people to participate in the Taichi movement, but also continue to enhance their physical quality enhancement.

\section{The research objects and methods}

This paper selects the development Taichi teaching college students as subjects, the first is to select two classes were divided into group $\mathrm{AB}$ control, test time is mainly arranged in the afternoon of the 14 points to 17 points, and ensure the room temperature of 20 degrees. Before a test can be performed to ensure adequate sleep, and the test is not subjective incommensurate circumstance. Acceptance test of the students 10 minutes rest in peace, when awake, you need to heart rate test, recording electrocardiogram under condition. There is then a recumbent 10 minutes rest, wait for the testing of students, breath is smooth, the body is fully relaxed state, then the experimental test. Tai Chi practice over time is approximately 5 minutes, respectively is 10 minutes sitting, supine, sitting. Then again repeat this exercise.

We can use the following formula to analyze the experiment.

$$
S D N N=\sqrt{\frac{1}{m} \sum_{i=1}^{m}\left(R R_{i}-\overline{R R}\right)^{2}}
$$


$m$ is a specific period of time after the total numerical, says $R_{i}$ is the $I$

RR interval, $\overline{R R}$ represents a specific time of normal RR interval .

$$
r M S S D=\sqrt{\frac{1}{m-1} \sum_{i=1}^{m-1}\left(R R_{i+1}-\overline{R R_{i}}\right)^{2}}
$$

Heart rate variability in the frequency domain analysis of main contains the TP value, LF value, $\mathrm{HF}$ value and LF/ value of $\mathrm{HF}$, see table 1.The former expresses the human autonomic nerve regulation function, if it was, then the human autonomic overall neurological function will be enhanced. LF is mainly a collection of sympathetic and vagal interaction link, when the total power output changes, then the corresponding reflecting sympathetic will also have corresponding change, while the HF value represents human vagus nerve ability level, finally LF said /HF is sympathetic and vagus nerve between the balance, if the ratio is higher, so the sympathetic nerve tension level will be higher. The frequency domain analysis index has a certain frequency range, and has the difference between them.

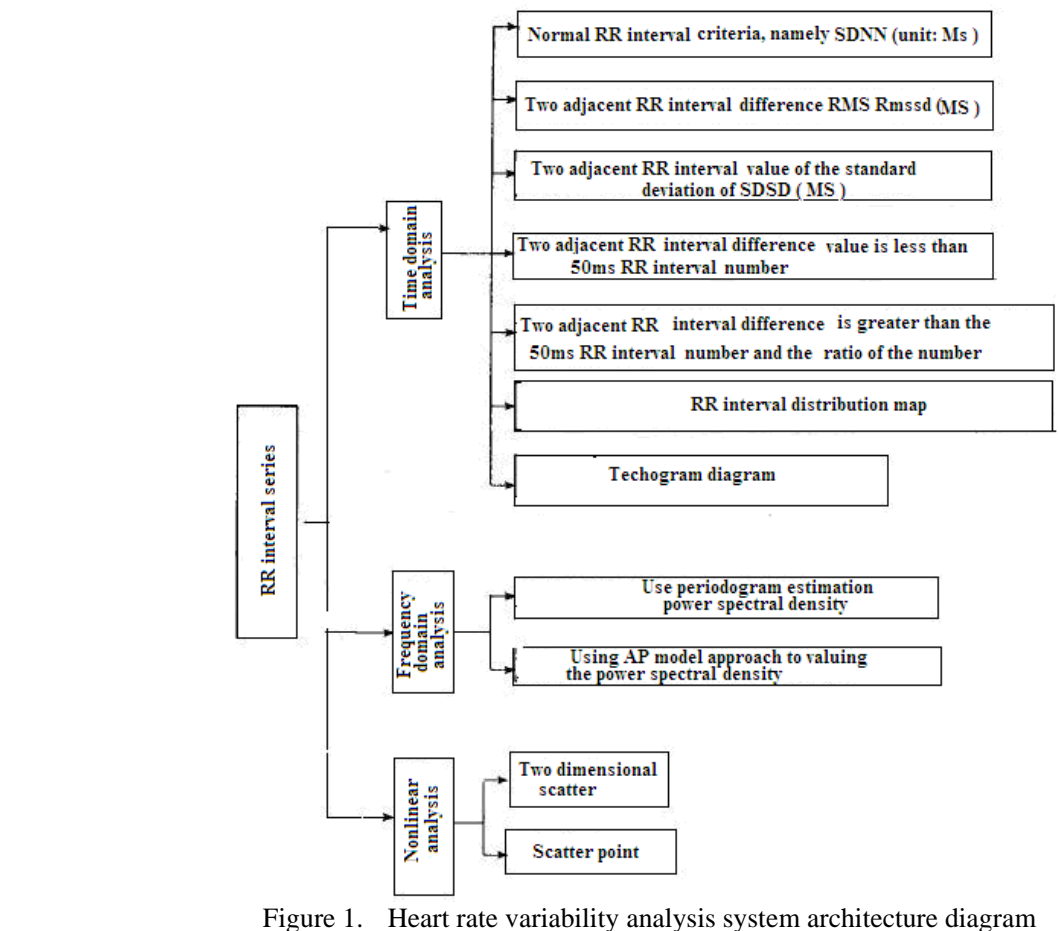

TABLE I. THE HEART RATE VARIABILITY IN THE FREQUENCY DOMAIN ANALYSIS OF RELEVANT INDICATORS

\begin{tabular}{|c|l|l|}
\hline Abbreviation & \multicolumn{1}{|c|}{ Full name } & $\begin{array}{c}\text { Frequency } \\
\text { range }\end{array}$ \\
\hline The value of $T \boldsymbol{P}$ & low frequency value & $0.03 \sim 0.42$ \\
\hline The value of $\mathbf{L F}$ & low frequency value & $0.03 \sim 0.16$ \\
\hline The value of $\mathbf{H F}$ & high frequency power & $0.16 \sim 0.42$ \\
\hline $\begin{array}{c}\text { The value of } \\
\mathbf{L F} / \mathbf{H F}\end{array}$ & $\begin{array}{l}\text { low frequency and high } \\
\text { frequency power ratio } \\
\text { value }\end{array}$ & \\
\hline
\end{tabular}

\section{Taichi special exercise on heart rate variability in the experimental analysis}

According to $\mathrm{AB}$ two groups of students to test data analysis, the mean RR interval calculated from the mean heart rate changes of values, see Table 2, AB two group interactions exist between differences. A group in a calm state at the time, the average heart rate value is greater than B group, in the state of motion, immediately, even after 1 minutes, 3 minutes and 10 minutes of state, group A values are smaller than the $\mathrm{B}$ group. 
TABLE II. MEAN RR INTERVAL CALCULATED FROM THE MEAN HEART RATE VARIATION

\begin{tabular}{|l|l|l|l|l|l|}
\hline & \multicolumn{1}{|c|}{$\begin{array}{c}\text { Calm } \\
\text { state }\end{array}$} & $\begin{array}{c}\text { Motion } \\
\text { state }\end{array}$ & Immediately & $\begin{array}{c}\text { 1 minutes after } \\
\text { exercise }\end{array}$ & $\begin{array}{c}\text { 10 minutes } \\
\text { after } \\
\text { exercise }\end{array}$ \\
\hline & & & & & \\
exercise
\end{tabular}

From the figure, we can be very intuitive to see, $A B$ two groups the average heart rate changes with motion and form curve in the state at the time, immediately, the average heart rate during exercise after the numerically largest, after the state average heart rate values showing a downward trend.

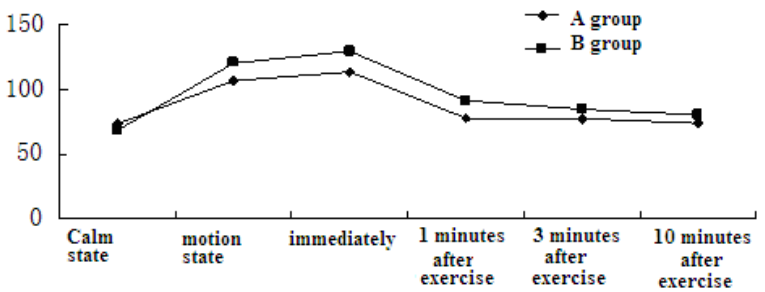

Figure 2. The mean RR interval calculated from the average heart rate variation

From the Figure 3 after experimental measurement standard deviation ( SDNN ) trend shows, group A standard deviation in any conditions is higher than those of B group value, but in a calm state, A group and B group, the standard deviation is the largest, while immediately state, the standard deviation is the smallest in the case of. And group AB standard deviation change trend are showing the first fall after a rise, rise state is relatively flat with some situation.

From Figure 4, the A group and B group and RMS is figure 3 standard deviation change toward roughly the same tendency. By the Figure 3, 4 may, in a calm state, the standard deviation ( SDNN ) and root mean square ( rMSSD ) are relatively high, but in the process of motion if reduced drastically, so follow-up after exercise, its recovery is relatively timely, but also for numerical in the relatively calm state a bit lower state.

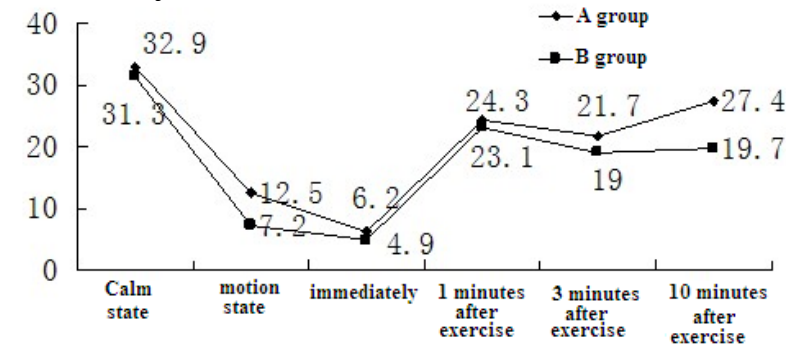

Figure 3. The experiment measuring the standard deviation ( SDNN ) changes and trends

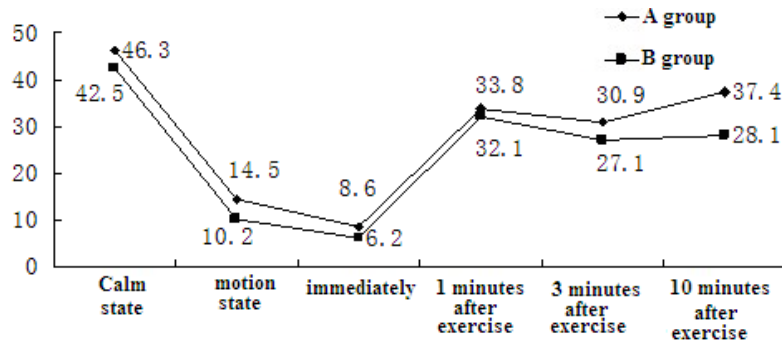

Figure 4. The experiment measurement of root mean square (rMSSD) changes and trends

From the Figure 5 after experimental measurement of sympathetic and vagal balance ( LF/HF ) of the B group, A group, a numerical case with figure 3 and Figure 4 of the standard deviation ( SDNN ) and root mean square ( rMSSD ) changes of sympathetic and vagal exactly the opposite, the balance is increased after the first drop the final equilibrium state, regional gentle. Group A sympathetic and vagal balance is always greater than B group relative to the state of numerical, but in a calm state, A, B group of sympathetic and vagal balance is relatively weak, in the state of motion, is balanced the strongest, most of numerical relativity.

\section{Conclusion}

In this test, fully considering the accuracy of measurement, so we create a relaxed atmosphere, so that the test can be completely relaxed, and can reflect the real test experiments on human autonomic nervous activity status. The research indicated that, in the quiet awake condition A groups in the total variability, RR interval, low frequency and high frequency parts are to be higher than that of B group, and $A B$ between two groups differences at the 0.05 level, no significant meaning, it also fully reflects the overall, human autonomic nerve function is in a state of relative balance. Taichi special 
sports can effectively reduce the heart rate variability, while long-term Taichi sport can not only make the increase of HR level can be reduced effectively, and can enhance the recovery rate and heart rate variability in a calm state independent of vagal nerve tension level, but also can effectively be plant of nervous system equilibrium level of ascension. So we need to fully recognize the Taichi sport for us to improve the physical quality, we will continue to strengthen the promotion and learning, so that it can better be the public to learn, to enhance people's physical quality level.

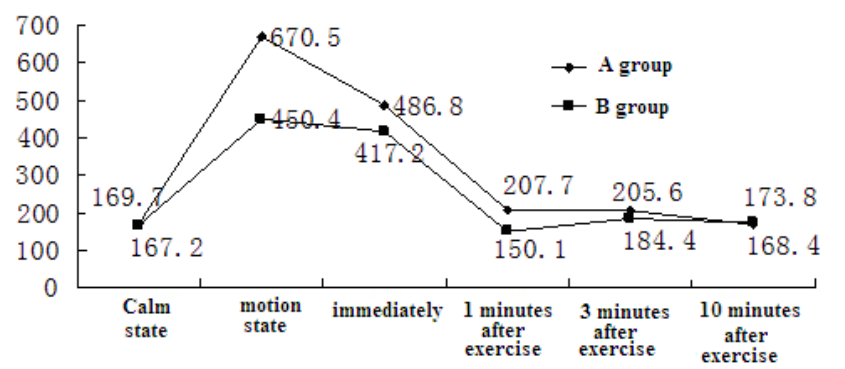

Figure 5. The experiment measurement of sympathetic and vagal balance ( LF/HF ) changes and trends

\section{References}

[1] Cao Lifeng,Zhao Jingguo.The character exercise to different postural position on heart rate variability.Biomedical Engineering Research,2010( 03):77-79.

[2] Sun Xusheng.The older people practice of tai chi in the analysis of heart rate variability. Journal of physical education,2012(2):67-69.

[3] Yang Yi. The movement to the health of the elderly on heart rate variability and its significance . Chinese Journal of sports medicine,2012(3):43-47.

[4] Liu Quan, Zhang Yong.24 Style Taichi Exercise on blood pressure, heart rate variability in different populations. Journal of Beijing Sport University,2011(12):24-28.

[5] Zhang Meizhen.The heart before and after exercise of female college students' body shape index HRV.2010 national exercise physiology of the papers set,2010:1021-1028

[6] Liu Xuexing incremental exercise and Competitive Aerobics Athletes in college students characteristics of heart rate variability.Jiangxi Normal University,2010:104-108.

[7] Xie Huihui.Tai Chi exercise on elderly depression and heart rate variability. Shanghai Institute of Physical Education,2011(4):63-67.

[8] Angie Hou.The basic Movement for women aged 25-35 exercise on heart rate variability. . Northeast Normal University, 2009(4):66-69.

[9] Zhang Xin.The exercise training on heart rate variability. Journal of Dalian University,2010(8):125-128.

[10] Zhao Jingguo.The incremental movement in the course of heart rate variability. Shandong university of science and technology,2009(03):23-25

[11] $\mathrm{Hu}$ Baiping.Taekwondo students special characteristics of heart rate variability. Journal of Anhwei Normal University,2010(10):225-227.

[12] Zhao Jingguo. Outstanding Sanda Athletes' heart rate variability. Chinese Journal of clinical rehabilitation, 2009 ( 4):59-63

[13] Zhang Lijuan. Quantitative physical exercise heart rate variability index variation characteristics. Chinese Journal of clinical rehabilitation,2010 ( 8):98-102 